MODELING, IDENTIFICATION AND CONTROL, 1993, VOL. 14, NO. 1, 3-26

doi:10.4173/mic.1993.1.1

\title{
Structured modeling of fish physiology
}

\author{
ODD A. OLSEN† and JENS G. BALCHEN†
}

Keywords: Metabolism, respiration, feeding behaviour, gills, kidneys, gastric evacuation, fish physiology, mathematical biology.

\begin{abstract}
The use of models in simulation and state estimation has proved useful in diverse applications, especially in industrial process control. The project presented here looked into the modeling of fish physiology for applications in fish physiology research and aquaculture. The models deal with gastric evacuation, metabolism, kidneys, gills, the cardiovascular system, and feeding behaviour and are based on data from the literature. Model responses are mostly in accord with real responses in principle, but, as with most models of complex biological systems, the numerical accuracy is low in several cases. However, these structured models enable researchers to test hypotheses by altering the submodels and parameters.
\end{abstract}

\section{Introduction}

Aquaculture is an ancient and important supplier of food in many countries, especially in Asia, where the fish are to a large extent left to themselves, often fed on household and agricultural leftovers. In several European and North American countries fish farming is becoming a significant industry. In this case the production is based on specially produced feeds and is both capital- and work-intensive. Because of the higher costs involved, the species cultured are those that get a high price on the market. A major species in Norway is salmon (Salmo salar). High costs also imply that the enterprises must be run efficiently.

First of all the fish must be fed the appropriate amounts of feed at the right times under varying environmental conditions that affect their appetite (temperature, oxygen level, light, season, etc.). If fed too little, the maximum growth rate will not be achieved. If fed too much, the excess feed will not be taken up by the fish and will be lost. In both cases plant efficiencies are lower than optimal; the latter case is also a serious source of local pollution. At present, feed wasted by overfeeding is estimated to cost Norwegian fish farmers about 100 million U.S. dollars a year, representing about $10 \%$ of the fish value.

Better understanding and knowledge of fish physiology and nutrition also enables the farmers to add other criteria to their optimal feeding strategies. Examples of this are given in Ruohonen $(1986,1988)$. The feeding strategy is in these cases adjusted by factors such as capital costs, predicted seasonal variations in temperature, oxygen level, and market price. This helps the farmer to control the growth rate of the fish to obtain the right weight at an optimal time of slaughter.

A fish farm manager's largest problem is to guess what the state of the fish really is. As the fish grow, the weight distribution becomes more and more uncertain. Fish cannot be weighed often because the handling induces stress that retards growth for days or even weeks. The total weight of fish kept in a pen is a compromise between capital cost and growth; if the fish density increases, the growth rate will decrease. A

Received 2 March 1992.

$\dagger$ Division of Engineering Cybernetics, Norwegian Institute of Technology, N-7034 Trondheim, Norway.

Reprinted with permission from Mathematical Biosciences, 1992, 112, 81-113. 
dense population is also more likely to develop diseases. By optimizing both feeding and densities, Norwegian fish farmers may save an estimated sum of 200 million U.S. dollars per year.

Visual observation of fish in sea pens is difficult, as is the interpretation of such observations. A fish farmer will probably not detect growth stagnation or altered behaviour by visual inspection, both factors being indicators of disease or environmental problems.

Experience has shown that the only instrument that in practice survives the harsh environment and retains a reasonable degree of accuracy is the thermometer. It is therefore clear that data for decision support are very limited.

One possible way to increase information about the state of a fish farm is to apply mathematical models of the fish and environment in combination with a state estimator and the available measurements (Olsen and Balchen 1988). Some measurements may be subjective and/or fuzzy, such as the observed feeding activity, which may be graded into, say four levels. Using such techniques, management-supporting systems may be realized for daily and long-term support. A more detailed discussion on instrumentation, information, and control technology applied to aquaculture can be found in Balchen (1989).

Fish research is another application of such models. Today most data are gathered from static experiments used for curve-fitting. The use of structured mathematical models, again combined with state estimation, may allow dynamic experiments, which give more information. The structured modeling approach will also enable the researcher to test different submodels, thus providing a tool for hypothesis testing. If high quality models are available, it will be possible to estimate states that are not directly measurable during the experiment.

In most cases, and certainly in the case of biology, it is not a question of choosing either modeling or empirical work. Rather, experimental work, data collection, and modeling are parts of an integrated process. The models may help in formulating knowledge and hypotheses in a suitable form, and data provide a basis for calibration and falsification of models or may indicate where the models can be improved. Structured models can be incrementally, and the submodels often independently, improved without having to discard the full model.

The purpose of this paper is to provide some basic models that we believe represent the most important relations in the areas of fish physiology treated.

\section{Gastric evacuation}

A prerequisite for optimal feeding is some knowledge about how the appetite of the fish varies in time, affecting the amount of feed that will be taken at a given instant. The appetite of the fish is strongly dependent on stomach filling (Elliott 1975, Grove, Loizides and Nott 1978); that is, after feeding, while the food is being digested and evacuated from the stomach into the intestines, the fish become increasingly motivated to approach and struggle for food. The fullness of the stomach is therefore an important state.

The evacuation rate has traditionally been modeled by a function of the type

$$
\frac{d V}{d t}=-c V^{b}
$$

where the constants $c$ and $b$ depend on factors such as temperature, feed properties, and fish weight, and $V$ is the volume of food remaining in the stomach after a meal. The 
value of the exponent $b$ is often chosen from assumptions about the digestive process, common values being $0,1,1 / 2$, and $2 / 3$ (Holmgren, Grove and Fletcher 1983, Jobling 1981). Such models describe the digestive process in the stomach, assuming that the digestive process is the limiting factor for the evacuation rate.

Several workers have found that the evacuation rate depends on the nutrient composition of the feed, energy-dense feed being evacuated at a lower rate than feed poor in nutrients. This suggests that the evacuation rate is controlled, possibly through the same hormonal and neural paths as in mammals. It would therefore be sound to model the digestive and evacuation processes separately. In aquaculture the feed usually consists of aggregates of finely minced particles; thus the digestive process taking place in the stomach is more rapid than when the fish prey on whole organisms. Hence, the evacuation process may be taken to be the limiting factor.

One may describe the evacuation rate as an intrinsic rate, assumed to be exponential, $b=1$ in (1), modulated by a feedback from chemoreceptors in the upper intestine (Jobling 1987). The objective of this control loop is probably to keep the nutrient flow rate slow enough for efficient nutrient absorption, yet evacuate poornutrient meals fast to yield space in the stomach for more food. The control mechanism found in many fish species is not perfect: as the nutrient density of the feed increases, the corresponding decrease in the evacuation rate levels out. This may be a result of the saturation of the control paths when the feed nutrient content is elevated above the level usually found in natural prey. The feeds used in aquaculture often have an energy content above $20 \mathrm{~kJ} / \mathrm{g}$, whereas natural prey usually lies in the range $4-8.5 \mathrm{~kJ} / \mathrm{g}$. The saturation effect may therefore be important in fish farming.

Our hypothesis for the evacuation rate control is that the flow is constant until the energy flow is equal to that given by the intrinsic function and that thereafter the control is ineffective and the emptying proceeds along the intrinsic pattern. After a meal of volume $V_{0}$, the stomach content thus initially decreases linearly with time, the emptying rate being $q_{m}$. Assuming the intrinsic function to be exponential, the intrinsic rate is $q_{i}=a V$. By equating the controlled and intrinsic rate,

$$
q_{m}=a V,
$$

and substituting for the remaining volume

the switchover time $t_{s}$ can be found:

$$
V=V_{0}-q_{m} t,
$$

$$
t_{s}=V_{0} / q_{m}-1 / a .
$$

The stomach volume at this time is $V_{s}=q_{m} / a$. The intrinsic emptying after $t_{s}$ yields the stomach volume

$$
V=V_{s} \exp \left(-a\left(t-t_{s}\right)\right) .
$$

The stomach may be defined as empty when a fraction $\beta V_{0}$ remains. Substitutions and rearrangement of the above equations then yield $\mathrm{s}$ the emptying time $t_{e}$ :

$$
t_{e}=\frac{V_{0}}{q_{m}}-\frac{1}{a}\left[1+\ln \left(\frac{a \beta V_{0}}{q_{m}}\right)\right] .
$$

This is valid for feeds that are high enough in energy content to demand controlled initial evacuation $\left(a V_{0}>q_{m}\right)$.

The published data on the evacuation rate are sparse and are often inconsistent. One reason for this is the measurement procedures usually applied. A number of fish 
are fed, fish are sampled and slaughtered at a few time intervals, and their stomach content is measured. It is not easy to find which is the influential control limiting function from these data. The rectangular hyperbola equation

$$
y_{e}=k C_{E} /\left(C_{E}+C_{50}\right)
$$

may be useful for modeling. Here, $C_{E}$ is the feed energy content and $C_{50}$ a parameter. Further, taking the set point for the controlled evacuation rate to be the inverse of this function $\left(q_{m}=1 / y_{e}\right)$, the resulting evacuation time delay due to the control can be derived as

$$
\Delta t=t_{e}-t_{e i}=\frac{V_{0} k C_{E}}{C_{E}+C_{50}}-\frac{1}{a}\left[1+\ln \left(\frac{a V_{0} k C_{E}}{C_{E}+C_{50}}\right)\right],
$$

where the intrinsic emptying time $t_{e i}=-\ln (\beta) / a$. This function is shown in Fig. 1 for a particular set of parameters: $k=2$ and $C_{50}=5 \mathrm{~kJ} / \mathrm{mL}$. The control of the flow may act through pulse modulation of the sphincter at the stomach outlet, as proposed in Jobling (1987).

By choosing the limiting function with appropriate parameters, the response can be fitted more accurately to a data set. However, the present model produces a response that in principle describes the observed saturation effect in energy content response. This indicates that the above hypothesis may be valid. However, more accurate methods for measuring evacuation rate are necessary to find out if real evacuation patterns follow an initial linear slope as postulated.

\section{Metabolism}

The nutrients absorbed from the intestines are transformed into energy, storage, and waste. Nutrients and waste are transported by the body fluids, and the transformations take place in the cells. Growth is mainly a result of stored matter.

The nutrients may be divided into amino acids, carbohydrates, and lipids. Each of these three groups behaves fundamentally differently through the metabolism, but the variations between the several species within each group are not so pronounced. It is therefore possible to aggregate the nutrients into the three main groups by using the mean properties of the species involved.

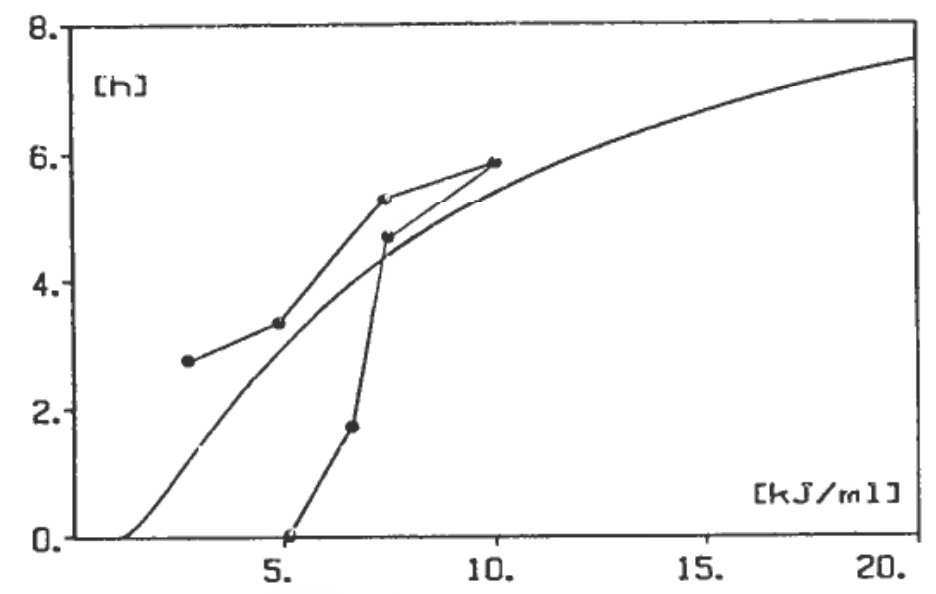

Figure 1. Energy concentration response of evacuation rate model. Dots: data calculated from Grove et al. (1978), using two kinds of feed diluters. 


\subsection{Metabolic stoichiometry}

The transformations taking place along the metabolic pathways can be described by chemical stoichiometric equations (Machiels and Henken 1986, Schulz, 1978). As an example, neglecting some insignificant parts, the overall equation for the total breakdown of amino acids can be written

$$
1 \mathrm{AA}+a \mathrm{O}_{2} \rightarrow b \mathrm{CO}_{2}+c \mathrm{NH}_{3}+d \mathrm{ATP},
$$

where the coefficients $a, b, c$, and $d$ depend on the actual amino acid (AA) composition. ATP is the energy yield in the form of adenosine triphosphate. ATP is the common denominator for energy used in the model. To simplify the relation of the model to measured data, all chemical equations are in grams per time unit, except for ATP, which is in moles per time unit. For a typical amino acid profile in fish, the coefficients in Eqn. (2) are $a=1 \cdot 26, b=1 \cdot 67, c=0 \cdot 16$, and $d=0.22$. Equivalent steps can be taken to develop the stoichiometric equations for the other metabolic paths in the model.

\subsection{Metabolic pathways}

What are believed to be the dominating metabolic paths were chosen for inclusion in the model. These constitute the basis for the formulation of state equations describing the level of nutrients under processing or transport through the body fluids or internally in the cells - the amino acid, carbohydrate, and lipid pools.

Feeds used in aquaculture are to a large extent made from less valuable fish, and the amino acid composition is almost equal in fish of different species. It is therefore not necessary to differentiate between amino acids stemming from the feed and those resulting from breakdown of body proteins. The amino acid paths incorporated into the model are

$$
\begin{array}{lll}
\text { Absorbed amino acid } & \rightarrow \text { amino acid pool } & \text { (subscript } A a \text { ), } \\
\text { Amino acid pool } & \rightarrow \text { energy } & \text { (subscript } a e \text { ), } \\
\text { Amino acid pool } & \rightarrow \text { glucose pool } & \text { (subscript } a g \text { ), } \\
\text { Amino acid pool } & \rightarrow \text { lipid pool } & \text { (subscript } a l \text { ), } \\
\text { Amino acid pool } & \leftrightarrow \text { stored protein } & \text { (subscript } a p \text { ). }
\end{array}
$$

The processing rates of these reactions are given by four rate controllers in the present model. The controller for stored protein rate may be negative when energy demands are larger than that provided by absorbed nutrients, representing a decreasing fish weight. A differential equation can be formulated for the amino acid pool state,

$$
\dot{x}_{A}=\left(w_{A a}-r_{a e} W-r_{a g} W-r_{a l} W-r_{a p} W\right) / V_{a}
$$

where the $r$ 's denote the process rates, $W$ the dry weight of the fish, $w$ the mass flow, and $V_{a}$ the equivalent reactor volume for amino acids, yielding the time constant of this state. The rates are believed to be mainly controlled by the concentration of hormones, which affects a number of cells taken to be proportional to the weight of the fish. The rates in the above equation therefore relate to the concentration of hormones.

Most carbohydrates are transformed into glucose when absorbed, and glucose is also the sole carbohydrate in the metabolic paths included in this model. Glucose is to some extent stored in the body as glycogen. The glycogen storage is mostly static in fish, decreasing only under hard physical stress, which is unlikely to occur for any significant duration in aquaculture. The glycogen storage is therefore not included in the model. Salmonids utilize carbohydrates poorly, and most of the glucose that is needed, mainly 
for the nervous system, is produced from amino acids. This is contrary to the mammalian case, where most glucose is produced from fat and glycogen. The paths participating in the glucose transformations are

$$
\begin{array}{lll}
\text { Absorbed glucose } \rightarrow \text { glucose pool } & \text { (subscript } A g \text { ), } \\
\text { Amino acid pool } & \rightarrow \text { glucose pool } & \text { (subscript } a g \text { ), } \\
\text { Glucose pool } & \rightarrow \text { lipid pool } & \text { (subscript } g l \text { ), } \\
\text { Glucose pool } & \rightarrow \text { energy } & \text { (subscript } g e \text { ). }
\end{array}
$$

The corresponding equation describing the glucose state is

$$
\dot{x}_{G}=\left(w_{A g}+0.59 w_{a g}-r_{g l} W-r_{g e} W\right) / V_{g} .
$$

$V_{g}$ is the glucose space for salmonids, about $14 \%$ of body volume. The flow of glucose produced from amino acids $\left(w_{a g}=r_{a g} W\right)$ is multiplied by a constant because less glucose is produced than the amount of amino acid that is consumed.

As was the case for amino acids, several types of lipids taking part in the metabolism are aggregated into a mean lipid representing the overall properties of the lipids. The lipid composition of the fish body is often taken to be correlated with the feed composition, and the variation in the average values for several types of feed may be small. Using such average values, the paths described by the model are

$$
\begin{array}{lll}
\text { Absorbed lipid } & \rightarrow \text { lipid pool } & \text { (subscript } A l), \\
\text { Glucose pool } & \rightarrow \text { lipid pool } & \text { (subscript } g l), \\
\text { Amino acid pool } & \rightarrow \text { lipid pool } & \text { (subscript } a l \text { ), } \\
\text { Lipid pool } & \rightarrow \text { energy } & \text { (subscript } l e \text { ), } \\
\text { Lipid pool } & \leftrightarrow \text { stored lipid } & \text { (subscript } l l \text { ). }
\end{array}
$$

The resulting differential equation for the lipid state is

$$
\dot{x}_{L}=\left(w_{A l}+0.36 w_{g l}+0 \cdot 22 w_{a l}-r_{l e} W-r_{l l} W\right) / V_{l},
$$

where $V_{l}$ represents the time constant of this state.

\subsection{Growth}

The growth in dry weight is described by the equations

$$
\dot{x}_{p}=0.86 w_{a p}=0.86 r_{a p} W,
$$

where the constant compensates for the water produced in the process, and

$$
\dot{x}_{l}=w_{l l}=r_{u} W .
$$

From this the dry weight gain is

$$
\dot{W}=\dot{x}_{p}+\dot{x}_{l} .
$$

The ash content of the fish must be added to this.

If the percentages of water $(m)$ and ash $(s)$ content of the fish are known, the growth rate of whole fish is

$$
\dot{W}_{w}=100\left(\dot{x}_{p}+\dot{x}_{l}\right) /(100-m-s) .
$$

Approximate values for $m$ and $s$ are $75 \%$ and $2.5 \%$, respectively, the former varying somewhat with feeding history and the ratio of body fat and protein (From and Rasmussen (1984). 
The terminal flows - oxygen and energy consumption, and carbon dioxide and ammonia production - can be described by arithmetic equations based on the stoichiometric equations. Thus, for the amino acid state, the terminal flows are

$$
\begin{aligned}
w_{\mathrm{O}_{2}} & =1.26 w_{a e}+0.53 w_{a g}+0.57 w_{a l}, \\
w_{\mathrm{CO}_{2}} & =1.67 w_{a e}+0.77 w_{a g}+0.16 w_{a b}, \\
w_{\mathrm{NH}_{3}} & =0.16 w_{a e}+0.16 w_{a g}+0.16 w_{a b},
\end{aligned}
$$

and

$$
w_{\mathrm{ATP}}=0 \cdot 22 w_{a e}+0 \cdot 77 w_{a g}-0.048 w_{a l}+\left\{\begin{array}{ll}
0 & \text { if } r_{a p}<0 \\
0.046 w_{a p} & \text { else }
\end{array} .\right.
$$

Similar equations can be derived for the other states, the sum of which yields the total terminal flows of the fish.

\subsection{Rate control}

The object of the rate control is to distribute nutrients and tune metabolic processes to provide and control the concentrations of metabolic intermediates and energy to support demand, maintain the fish fit for survival, and invest excess nutrients into growth.

In a real fish the rates are controlled by hormone concentrations. Most knowledge about hormone activation and responses derives from experiments on mammals, especially the white rat. Fish and mammals are distantly related on the evolutionary tree, and one cannot assume that the same relations exist in fish as in mammals. The knowledge on fish hormone mechanisms is sparse and to some extent contradictory. The strategy for this modeling was therefore not to model the hormonal processes directly, but to apply controllers that simulate the known cause-and-effect systems of metabolic control of real fish.

Energy production in the fish mainly supports food processing and osmotic and mechanical work. The total energy is often divided into routine and feeding metabolism. Routine metabolism is that of an active, nonfeeding, and nonstressed fish, given in From and Rasmussen (1984) as equivalent weight loss

$$
\dot{W}_{w}=154 \times 10^{-6} \exp (0.0875 T) W_{w}^{0.7688} .
$$

The fish wet weight loss $\dot{W}_{w}$ is in grams per hour and must be recalculated into moles of ATP per hour, assuming a protein and lipid mobilization ratio as in Eqns. (4) and (5). Feeding respiration is the energy expenditure for digestion and metabolic processing of the nutrients after a meal. Neglecting digestion costs, this follows from the stoichiometric equations along the line exemplified in Eqn. (3).

The energy supplied by glucose is used mainly for the neural system. Based on few data, a reasonable function for this rate is

$$
r_{g e}=8.4 \times 10^{-6} \exp (0.069 T)
$$

where $T$ is the temperature in ${ }^{\circ} \mathrm{C}$.

Except for the neural requirements for glucose, the rest of the routine energy and feeding energy is assumed to be supplied by amino acids and lipids. In mammals more energy is provided by fat than by amino acids, the opposite being the case for salmonids. Measurements have shown that protein may provide $80-90 \%$ of the energy 
supply. The proportion of energy provided by amino acids can be represented by a variable $r \in[0,1]$, and the fat and amino acid combustion rates can be described by

$$
r_{a e}=r w_{\text {ATP }} / 0 \cdot 22
$$

and

$$
r_{l e}=(1-r) w_{\mathrm{ATP}} / 0.51,
$$

where $w_{\text {ATP }}$ is the total energy demand minus the energy provided by glucose, and the constants are the energy yield (moles of ATP per gram).

It is fair to suggest that the energy mobilization ratio $r$ depends on the state of the fish, for example, the body protein/lipid ratio (analogous with the lipostatic theory of CNS control of feed intake)

$$
r=f^{*}\left(x_{p}, x_{l}\right),
$$

where $f^{*}$ is a suitable function. Several sources of data show that the $x_{l} / x_{p}$ ratio in fish rises with increasing supply of feed and increasing level of lipids in the feed. The dependency on feeding level $(f \in[0,1])$ can be modeled by the straight line

$$
L_{r f}=a_{f}+b_{f} f .
$$

The response from the fat/protein ratio in the feed $\left(L_{f} / P_{f}<1\right)$ to fat/protein ratio in the body can be described by

$$
L_{r l}=g_{f}\left(\frac{L_{f}}{P_{f}}-d_{f}\right) \frac{L_{r f}}{a_{f}+b_{f}} .
$$

This function is more influential when the feeding level is high. The sum $L_{r}=L_{r f}+L_{r l}$ is now used as a reference for the energy allocation ratio controller,

$$
r=-k_{l p}\left(x_{l} / W-L_{r}\right)+0 \cdot 5,
$$

where $k_{t p}$ is the controller gain, $L_{r}$ the controller set point, and the last term a controller output bias. All parameters in the above equations must be found from experimental data.

The qualitative or quantitative properties of lipogenesis from amino acids are not well known for fish. One assumption that may be valid is that lipids are produced if there are excessive amino acids. If the amino acid excess is denoted by $C_{a x}$, the control functions for protein storage and lipid production can be postulated as

$$
\begin{aligned}
C_{a x} & =k_{a x}\left(x_{A}-A_{r}\right), \\
r_{x} & = \begin{cases}a_{x}+b_{x} r & \text { if } C_{a x}>0 \\
0 & \text { else, }\end{cases} \\
r_{a p} & =C_{a x}\left(1-r_{x}\right), \\
r_{a l} & =C_{a x} r_{x} .
\end{aligned}
$$

Here $A_{r}$ is a postulated amino acid reference level. The parameters $a_{x}$ and $b_{x}$ are unknown, but reasonable guesses may be 0 and 0.4 , respectively. When $r$ is high, corresponding to the case when most of the energy demand is provided by amino acids, a major part of the amino acid excess is transformed into lipid. 
Gluconeogenesis has been observed to decrease if exogenous glucose is supplied. This indicates that gluconeogenesis is under the control of the blood glucose level:

$$
r_{a g}= \begin{cases}-k_{a g}\left(x_{G}-G_{r}\right) & \text { if } G_{r}>x_{G} \\ 0 & \text { else, }\end{cases}
$$

where $G_{r}$ is a postulated glucose reference level and $k_{a g}$ the controller gain. When the glucose level is higher than the reference, excess glucose may be removed by the production of lipids, independent of the actual lipid level:

$$
r_{g l}= \begin{cases}k_{g l}\left(x_{G}-G_{r}\right) & \text { when } x_{G}>G_{r} \\ 0 & \text { else. }\end{cases}
$$

The storage of lipids in the body may be controlled from the lipid level deviation from the reference $L_{r}$ :

$$
r_{u}=k_{u}\left(x_{L}-L_{r}\right)
$$

where $k_{l l}$ is the controller gain.

\subsection{Model simulation}

The above model was calibrated using data compiled from several sources, mainly based on salmonids. Most parameters for the controllers were adjusted to get sound simulated responses; data on dynamic metabolic responses in fish are virtually nonexistent. However, many parameters of the static relations are obtained from the chemical equations and are thus fairly accurately determined. The parameters used are given in Table 1.

Figure 2 illustrates a few responses of the model compared with analogous experimental results on oxygen consumption and nitrogen production. In this case an intrinsic evacuation rate, $b=1$ and $c=0 \cdot 15 \mathrm{~h}^{-1}$ in Eqn. (1), was used. The experimental data are from fish fed every morning; therefore the rise in oxygen consumption before feeding may be caused by increased physical activity due to the feed expectations of the fish combined with increased activity at sunrise.

Responses of the internal states of this model cannot presently be verified by experimental data, but responses not shown here demonstrate that the model behaves qualitatively as expected. Owing to lack of relevant data one can only conclude that the model performs reasonably well with respect to both quality and numerical values compared to data on oxygen consumption and $\mathrm{NH}_{3}$ excretion.

\begin{tabular}{lrlllc}
\hline$W$ & $29 \mathrm{~g}$ & $L_{r}$ & $50 \mathrm{mg} / 100 \mathrm{~mL}$ & $a_{x}$ & $0-4$ \\
$V_{a}$ & $5 \%$ & $G_{r}$ & $60 \mathrm{mg} / 100 \mathrm{~mL}$ & $b_{x}$ & $0-0$ \\
$V_{g}$ & $14 \%$ & $A_{r}$ & $30 \mathrm{mg} / 100 \mathrm{~mL}$ & $a_{f}$ & 10 \\
$V_{l}$ & $5 \%$ & $k_{a x}$ & 0.025 & $b_{f}$ & 20 \\
$m$ & $75 \%$ & $k_{a g}$ & 0.01 & $g_{f}$ & 21 \\
$s$ & $2 \cdot 5 \%$ & $k_{g l}$ & 0.022 & $d_{f}$ & 0.2 \\
$L_{f}$ & $6.4 \%$ & $k_{l p}$ & 0.5 & $T$ & $15^{\circ} \mathrm{C}$ \\
$P_{f}$ & $33 \%$ & $k_{l l}$ & 0.05 & & \\
$C_{f}$ & $15 \%$ & $k_{r x}$ & 2.0 & & \\
\hline
\end{tabular}

Table 1. Parameters used in the metabolic model for the simulations of fig. 2. 

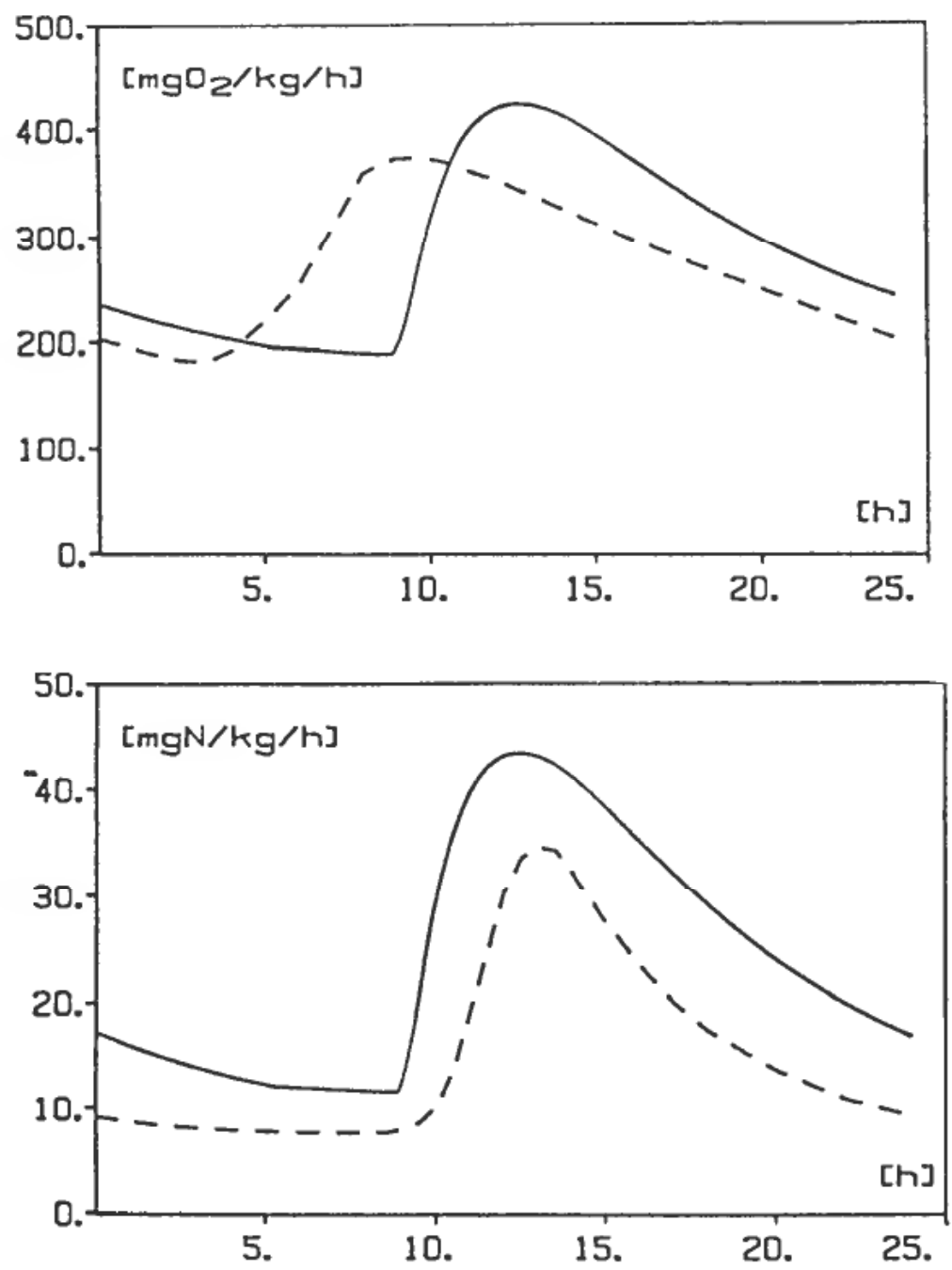

Figure 2. Respiration of $29 \mathrm{~g}$ fish at $15^{\circ} \mathrm{C}$ fed a $0-85 \mathrm{~g}$ meal from $8: 30$ to 9:30 (top) and nitrogen excretion of same fish (bottom). Solid lines: simulated; broken lines: data from Brett and Groves (1979).

\section{Kidneys}

It is known that several species of carnivorous fish (e.g., the salmonids) are not able to control their blood concentration of glucose well when given large doses of glucose. Although insulin affects the blood level of glucose in these fishes, insulin in usually not produced in large enough quantities to handle glucose overloads. This resembles the situation in mammals with type 2 diabetes. The upper stationary limit of blood glucose level in diabetic mammals is given by the kidney threshold, reflecting the glucose concentration above which blood glucose is lost through the kidneys. If this also is a path for glucose loss in fish following ingestion of the carbohydrate-rich feeds given in aquaculture, it may be useful to add a model to this to the metabolic model. 


\subsection{Glucose filtration}

The kidney nephrons mainly consist of a glomerular capsule followed by a tubule. When blood enters the kidney, the plasma and its smaller molecules are filtered through the walls of the glomerular capillaries into the capsule. The concentration of glucose in the capsule is equal to that of the blood. The total rate of plasma flow into all the capsules of the kidneys is called the glomerular filtration rate (GFR). Then the filtrate flows through the tubule and is mostly reabsorbed into blood capillaries. The part that is not reabsorbed forms the urine.

The reabsorption of glucose is active, and this transport is saturated at the glucose transport maximum $\left(\mathrm{T}_{M}\right)$. When the glucose rate in the filtrate exceeds this limit, glucose will be lost through the urine.

Nephrons in the teleostian kidney are functionally equal to those of mammals, but in several species $T_{M}$ has been found to vary linearly with the filtration rate. This indicates that a varying number of nephrons in the kidney are activated in an on/off fashion depending on the total filtration rate rather than the variation of the filtration rate of each nephron. In freshwater-adapted trout, about $50 \%$ of the nephrons are filtering, whereas in seawater the filtering population is less than $10 \%$, reflecting the different needs for osmoregulation.

\subsection{Model}

For each nephron $n$ in the filtering population $N$, the mass flow of filtered glucose is

$$
w_{g f n}=q_{f n} C_{g b},
$$

where $q_{f n}$ is the filtrate flow and $C_{g b}$ the blood glucose concentration. If the filtration rate is assumed to be equal in all nephrons (on/off control), the total filtration rate in the kidney is

$$
w_{g f}=N q_{f n} C_{g b}=q_{f} C_{g b},
$$

where $q_{f}$ is the total glomerular filtration rate.

The reabsorbed glucose mass flow for a single nephron is

$$
w_{g r n}= \begin{cases}w_{g f n} & \text { if } w_{g f n}<T_{M m}, \\ T_{M n} & \text { otherwise, }\end{cases}
$$

where $T_{M n}$ is the kidney threshold for a single nephron. If the reabsorption saturation level is assumed to be equal for all nephrons, the total reabsorption capacity is

$$
T_{M}=N T_{M n}
$$

Therefore, because of the varying degree of nephron recruitment $(N)$ found in fish, the glucose threshold is variable and not constant as in mammals. From the above, the total reabsorption is

$$
w_{g r}= \begin{cases}w_{g f} & \text { if } w_{g f}<T_{M}, \\ T_{M} & \text { else. }\end{cases}
$$

The glucose loss through the urine is the difference between that filtered and that reabsorbed:

$$
w_{g u}=w_{g f}-w_{g r}= \begin{cases}0 & \text { if } w_{g f}<T_{M}, \\ q_{f} C_{g b}-T_{M} & \text { else. }\end{cases}
$$


From this the condition for total reabsorption of glucose is found to be

$$
q_{f} C_{g b}<T_{M},
$$

and the kidney threshold is

$$
C_{g b t}=T_{M} / q_{f}
$$

\subsection{Comparison with real data}

Few data are available, and the following calculations are only intended to indicate the order of values. The values for the transport maximum and filtration rate as given in Table 2 are taken from Kenyon, McKeever, Oliver and Henderson (1985), based on experiments with freshwater- and seawater-adapted 200-300 g rainbow trout (Oncorhynchus mykiss, formerly Salmo gairdneri). The corresponding values for the kidney threshold were calculated according to the above model. From these data one would expect the blood glucose level to be higher in fish adapted to seawater than in those adapted to freshwater when fed high-carbohydrate diets. Data on glucose level vs. feed carbohydrate content were not available. But data from Sundby, Eliassen, Blom and Aasgaard (1991) on fish fed commercial diets may be indicative: The blood glucose level in rainbow trout in freshwater and Atlantic salmon in seawater was $80-110 \mathrm{mg} / 100 \mathrm{~mL}$ and $115-140 \mathrm{mg} / 100 \mathrm{~mL}$, respectively. However, these data neither support nor contradict the above hypothesis, because these differences may be due to other factors.

\section{Circulation and ventilation}

An important limiting factor for activity and metabolism is the ability of the fish to extract oxygen from the water and excrete $\mathrm{CO}_{2}$ into the water. Although some exchange can occur through the skin, practically all exchange in the salmonids happens through the gills. The blood flow of teleost fish differs from the mammalian as the fish have only one circuit through the heart. Gills are therefore in series with the vascular bed of the tissues. The gills are perfused with ventral arterial blood, and the gas exchange over the gill membrane is by passive diffusion for both $\mathrm{CO}_{2}$ and $\mathrm{O}_{2}$. The dorsal arterial blood from the gills carries the oxygen to the tissues, where $\mathrm{CO}_{2}$ is loaded. The venous blood is then pumped back to the gills, where $\mathrm{CO}_{2}$ is unloaded. Water has a large capacity to absorb carbon dioxide, and it is therefore the extraction of oxygen that is the limiting factor for fish. The following model of the cardiovascular system therefore neglects $\mathrm{CO}_{2}$.

\subsection{Haemodynamics}

The blood flow is pulsatile, and pressure pulses are therefore reflected from geometric irregularities and bifurcations along the blood vessels. For all but the largest fish, for example, tuna, such transmission line effects are not significant. Neglecting

\begin{tabular}{cccc}
\hline Adaptation & $\begin{array}{c}T_{M} \\
{[\mu \mathrm{g} /(\mathrm{kg} / \mathrm{min})]}\end{array}$ & $\begin{array}{c}q_{f} \\
{[\mu \mathrm{L} /(\mathrm{kg} / \mathrm{min})]}\end{array}$ & $\begin{array}{c}C_{\text {gbt }} \\
(\mathrm{mg} / 100 \mathrm{~mL})\end{array}$ \\
\hline FW & 190 & 115 & 165 \\
SW & 65 & 18 & 360
\end{tabular}

Table 2. Transport maximum for glucose and glomerular filtration rate in saltwater- and freshwater-adapted rainbow trout (Kenyon et al.), and calculated kidney threshold. 
pulsatile components of the flow, the vascular system can be modeled as two resistances in series, the gill and peripheral (tissue) flow resistances, the flow being driven by the heart ventral pressure. In this case the dorsal aorta pressure (the pressure over the tissues) is

$$
p_{d a}=p_{v a} R_{p} /\left(R_{p}+R_{g}\right)
$$

where $p_{v a}$ is the ventral aorta pressure (the pressure over the heart), and $R_{p}$ and $R_{g}$ are the peripheral and gill flow resistances, respectively. By the same model, the blood flow is given by

$$
q_{b}=p_{v a} /\left(R_{p}+R_{g}\right) \text {. }
$$

Both flow resistances may be under hormonal and central and local nervous control, but the gill resistance has been found to be nearly constant.

\subsection{Blood}

Oxygen is transported both in physical solution in the plasma and chemically bound in the haemoglobin of the red blood cells. The degree of oxygen saturation $(S)$ of haemoglobin as a function of oxygen partial pressure $\left(p_{b}\right)$ is given approximately by Hill's equation

$$
S=p_{b}^{n} /\left(p_{b}^{n}+p_{50}^{n}\right),
$$

where $p_{50}$ is the half-saturation pressure and $n$ is a parameter that for trout has a maximum of 2.0 at $\mathbf{p H}=7.5$. The carrying capacity for oxygen is dependent on such factors as temperature and $\mathrm{pH}\left(\mathrm{CO}_{2}\right.$ concentration). A better approximation of the blood properties for both $\mathrm{O}_{2}$ and $\mathrm{CO}_{2}$ can be found in Malte and Weber (1985); however, the uncertainties in the parameters involved does not improve the accuracy of the model over that based on Hill's equation.

When the parameters for plasma and haemoglobin properties are known, the concentration of oxygen in the blood can be found:

$$
C_{b}=\gamma p_{b}+4 C_{\mathrm{Hb}} p_{b}^{n} /\left(p_{b}^{n}+p_{50}^{n}\right),
$$

where $\gamma$ is the solubility in plasma and $C_{\mathrm{Hb}}$ is the concentration of haemoglobin, each molecule being able to bind four $\mathrm{O}_{2}$ molecules. The oxygen carried in the physical solution in the plasma can be neglected because the dominant oxygen transport is by haemoglobin.

\subsection{Gills}

The filaments of the gills are positioned to let the blood flow in the direction opposite to the water at their interface. This allows very efficient gas transfer. Since the gas exchange is passive, the gills may be modeled as a passive countercurrent gas exchanger. The oxygen partial presssure of the water $\left(p_{w}\right)$ and blood $\left(p_{b}\right)$ can thus be described by a pair of partial differential equations,

$$
\frac{\partial p_{w}}{\partial z}=\frac{D\left[p_{w}(z)-p_{b}(z)\right]}{q_{w} \alpha}
$$

and

$$
\frac{\partial p_{b}}{\partial z}=\frac{D\left[p_{w}(z)-p_{b}(z)\right]}{q_{b} \beta}
$$


where $z \in[0,1]$ is the distance from the ventral inlet along the exchanger, $q_{w}$ the water flow, and $D$ the apparent diffusion coefficient. $\alpha$ and $\beta$ are the solubilities $(d C / d p)$ of oxygen in water and whole blood, respectively. $\beta$ is generally a nonlinear function varying along the gill filament with the $\mathrm{O}_{2}$ concentration, obtained by derivation of Eqn. (11). In addition to this, Hill's equation may be modified by the Bohr shift: the slope of the dissociation curve increases with increasing $\mathrm{pH}$. This effect requires the $\mathrm{pH}$, and therefore also the concentration of $\mathrm{CO}_{2}$, to be included in the model. A derivation of a complete set of partial differential equations describing the $\mathrm{O}_{2}$ and $\mathrm{CO}_{2}$ exchange over the gills, including the nonlinear Hill's equation and Bohr shift, can be found in Malte and Weber (1985). These nonlinear partial differential equations represent a twopoint boundary value problem and can be solved by numerical integration (e.g., by the 'shooting' method (Press, Flannery, Teukolsky and Vetterling, 1989)).

In the following model a constant $\beta=0.5 C_{\max } / p_{50}$ will be used, thus drastically reducing the computational burden. The nonlinear model will typically yield about $20 \%$ more oxygen uptake than the linear one. However, in the following model, feedback controllers are assumed to compensate for this inaccuracy together with the uncertainty of the diffusion coefficient. A linear approximation is therefore valid, at least in this case where qualitative modeling is most important.

The partial differential equations can therefore be solved analytically, and the water transfer efficiency can be derived (Piiper 1982, Piiper and Scheid 1984):

$$
\eta=\left(p_{I}-p_{E}\right) /\left(p_{I}-p_{v}\right)=(1-\exp (-Z)) /(X-\exp (-Z)),
$$

where

$$
X=\frac{q_{w} \alpha}{q_{b} \beta} \text { and } Z=D \frac{1-1 / X}{q_{b} \beta}
$$

and $p_{I}, p_{E}$, and $p_{v}$ are the partial oxygen pressures of the inspired and expired water and ventral blood at the gill inlet, respectively.

\subsection{Model}

A single-controller model for the cardiovascular/respiratory system was presented in Taylor, Houston and Horgan (1968) and, although behaving well, was apparently tested only on the data used for model calibration. The following model is a development of that model and consists of two states: the oxygen concentration of the blood at the gill (dorsal arterial) $C_{a}$ and tissue (venous) $C_{v}$ outlets. The differential equation describing the first state is

$$
\dot{C}_{a}=\left[q_{b}\left(C_{v}-C_{a}\right)+w_{g}\right] / V_{g},
$$

where $V_{g}$ is the apparent volume of the gill vascular bed. The oxygen transfer from the water is given by

$$
w_{g}=\left(p_{I}-p_{E}\right) q_{w} \alpha
$$

Applying Eqn. (14) yields

$$
w_{g}=\frac{1-\exp (-Z)}{X-\exp (-Z)}\left(p_{I}-p_{v}\right) q_{w} \alpha .
$$

The state of the venous blood is given by

$$
\dot{C}_{v}=\left[q_{h}\left(C_{a}-C_{v}\right)-w_{t}\right] / V_{s},
$$


where $V_{s}$ is the apparent volume of the tissue vascular bed and $w_{t}$ the flow of oxygen from the blood into the tissues.

Arterial and venous blood oxygen partial pressures are given by the dissociation curve of the blood, approximated by a rearrangement of Hill's equation (10),

$$
\begin{aligned}
& p_{a}=p_{50}\left[S_{a} /\left(1-S_{a}\right)\right]^{1 / n}, \\
& p_{v}=p_{50}\left[S_{v} /\left(1-S_{v}\right)\right]^{1 / n},
\end{aligned}
$$

where $S_{a}=C_{a} / C_{\max }$ and $S_{v}=C_{v} / C_{\max }$. In this case the nonlinearity of Hill's equation must be included because the concentration/pressure relation is an important factor in the model and cannot be compensated for by the controllers.

\subsection{Control structure}

The variables to be controlled are blood flow, water flow, blood pressure, tissue flow resistance, gill diffusion coefficient, and venous and arterial oxygen concentrations.

The peripheral resistance depends on the parallel combination of the individual capillary resistances. These are under independent local control in an on/off fashion by the concentration of metabolites in their vicinity. Put in other words, they independently open when perfusion is needed. Relative to the simplifications in this model, this means that the peripheral resistance adjusts itself according to the metabolic oxygen demand. A controller may simulate this, adjusting the blood flow by operating on the peripheral resistance; the aim of this is to maintain the venous oxygen concentration at the set point $C_{v r}$,

$$
q_{b}=q_{b 0}+k_{1}\left(C_{v r}-C_{v}\right) \text {. }
$$

This assumes that no nervous control is present, which is valid for mammals under normal conditions of stress.

The apparent diffusion coefficient of the gills may be controlled by hormonal means, but there is a strong correlation between the pressure drop over the gills and the diffusion coefficient. This is probably due to a distribution of the pressure needed to open the individual filaments for blood perfusion, so when the pressure drop increases, more and more filaments open. This can be modeled as a function of the ventral and dorsal blood pressures,

$$
D=D_{0}+k_{2}\left(p_{v a}-p_{d a}\right)
$$

The dorsal pressure is given by

$$
p_{d a}=p_{v a}-R_{g} q_{b}
$$

where $R_{g}$ is taken to be constant.

The ventral blood pressure provided by the heart increases during exercise. This may be, and is modeled as being, controlled by the deviation of the arterial oxygen content from the set point $C_{a r}$,

$$
p_{v a}=p_{v a 0}+k_{3}\left(C_{a r}-C_{a}\right) \text {. }
$$

The oxygen sensor is presumably located near the gills, but the precise location is not known.

The ratio of the blood and water flows is often assumed to be about 1:10, independent of exercise level. However, some experiments show this ratio to increase/decrease by a factor of 0.6-1.4 from rest to maximum exercise. This ratio is not 
measured directly and is therefore dependent on the accuracies of the data and assumptions involved in the calculations. Preliminary experiments with the present model indicated that a ratio increase by a factor of 2 was necessary to yield good simulation results at all levels of exercise. Our hypothesis is that this ratio $r$ is controlled by the deviation of the arterial blood oxygen concentration from a reference level,

$$
r=r_{0}+k_{4}\left(C_{a r}-C_{a}\right) \text {. }
$$

The resulting water flow is then

$$
q_{w}=r q_{b}=\left[r_{0}+k_{4}\left(C_{a r}-C_{a}\right)\right] q_{b} .
$$

This implies that the ventral blood pressure and water flow act in parallel to increase oxygen uptake.

\subsection{Model simulation}

The model was calibrated with data from several sources. Temperature is an important parameter for the metabolic rate, the solubility of oxygen in water and blood, and the blood properties. This was not implemented in the model, so nominal values were used, mostly based on data from experiments in the $10-15^{\circ} \mathrm{C}$ range.

The oxygen consumption was taken to be (Kiceniuk and Jones, 1977)

$$
w_{t}=10^{1 \cdot 27+U / U_{m}} \mu \mathrm{mol} /(\mathrm{kg} / \mathrm{min}),
$$

where $U_{m}$ is the maximum speed for sustained swimming and is described by

$$
U_{m}=12 \cdot 4 L^{0.55} \mathrm{~cm} / \mathrm{s},
$$

$L$ being the body length in centimetres.

\begin{tabular}{|c|c|c|c|c|c|}
\hline \multirow[b]{2}{*}{ Variable } & \multicolumn{2}{|c|}{ Rest } & \multicolumn{2}{|c|}{ Max. exercise } & \multirow[b]{2}{*}{ Units } \\
\hline & Ref. data & Model & Ref. data & Model & \\
\hline$C_{v}$ & $3 \cdot 17$ & $3 \cdot 20$ & 0.6 & 0.61 & $\mathrm{mmol} / \mathrm{L}$ \\
\hline$C_{a}$ & $4 \cdot 64$ & $4 \cdot 65$ & $4 \cdot 33$ & $4 \cdot 22$ & $\mathrm{mmol} / \mathrm{L}$ \\
\hline$p_{v}$ & $4 \cdot 43$ & $4 \cdot 52$ & $2 \cdot 13$ & 1.22 & $\mathbf{k P a}$ \\
\hline$p_{a}$ & $18 \cdot 27$ & $17 \cdot 9$ & $16 \cdot 8$ & $8 \cdot 6$ & $\mathbf{k P a}$ \\
\hline$q_{t}$ & 25 & 25 & $193 \cdot 7$ & 184 & $\mu \mathrm{mol} / \mathrm{min}$ \\
\hline$q_{b}$ & $17 \cdot 6$ & $17 \cdot 2$ & $52 \cdot 6$ & $50 \cdot 9$ & $\mathrm{~mL} / \mathrm{min}$ \\
\hline$q_{w}$ & 211 & 207 & $<1700$ & 1485 & $\mathrm{~mL} / \mathrm{min}$ \\
\hline$p_{v a}$ & 4.7 & 4.75 & $7 \cdot 2$ & $7 \cdot 58$ & $\mathbf{k P a}$ \\
\hline$p_{d a}$ & $3 \cdot 8$ & $3 \cdot 71$ & $4 \cdot 5$ & $4 \cdot 53$ & $\mathbf{k P a}$ \\
\hline$R_{t}$ & $0-22$ & 0.215 & 0.085 & $0-089$ & $\mathrm{kPa} \min / \mathrm{mL}$ \\
\hline$q_{w} / q_{b}$ & & $12 \cdot 0$ & & $29 \cdot 2$ & \\
\hline$n$ & & $2 \cdot 0$ & & $2 \cdot 0$ & \\
\hline$C_{\max }$ & & $4 \cdot 8$ & & $4 \cdot 8$ & $\mathrm{mmol} / \mathrm{L}$ \\
\hline$p_{50}$ & & $3 \cdot 2$ & & $3 \cdot 2$ & $\mathbf{k P a}$ \\
\hline$p_{E}$ & & $12 \cdot 4$ & & $12 \cdot 3$ & $\mathbf{k P a}$ \\
\hline$D$ & & $2 \cdot 4$ & & 14.9 & \\
\hline
\end{tabular}

Some parameters were tuned by comparing simulation runs with experimental data from static experiments, that is, experiments where the fish were exercised at a constant level (Kiceniuk and Jones, 1977). The results are summarized in Table 3. Although the

Table 3. Comparison of data from static experiments (Kiceniuk and Jones, 1977) (ref. data) and present model at minimum and maximum exercise. 
agreement between model and real data is good, a significant deviation exists for the partial pressures at maximum exercise. This is probably caused by a shift of the blood properties at increasing load (e.g., Bohr shift caused by increased levels of $\mathrm{CO}_{2}$ and decreased $\mathrm{pH}$ ), which is not taken into account by Hill's equation.

Following the calibration phase, the transient behaviour of the model was compared to dynamic experimental data (Stevens and Randall $1967 \mathrm{a}, \mathrm{b}$ ). In this experiment the exercise was gradually increased from a low level to a high level, kept at this level, and then lowered again, each phase taking $5 \mathrm{~min}$. An estimate of the corresponding oxygen consumption rate function was given in Taylor $e t$ al. (1968), and this function was used for the simulations.

Figure 3 shows the results for the blood pressure and Figure 4 shows the oxygen partial pressure. The simulated blood pressure responses agree well with the real data, but they indicate that the oxygen consumption rate function applied lasts too long after the end of exercise. The shapes of both the arterial and venous blood oxygen pressures are also correlated to some degree with the model responses. The arterial oxygen pressure in both cases increases at the beginning of the active interval, decreases during
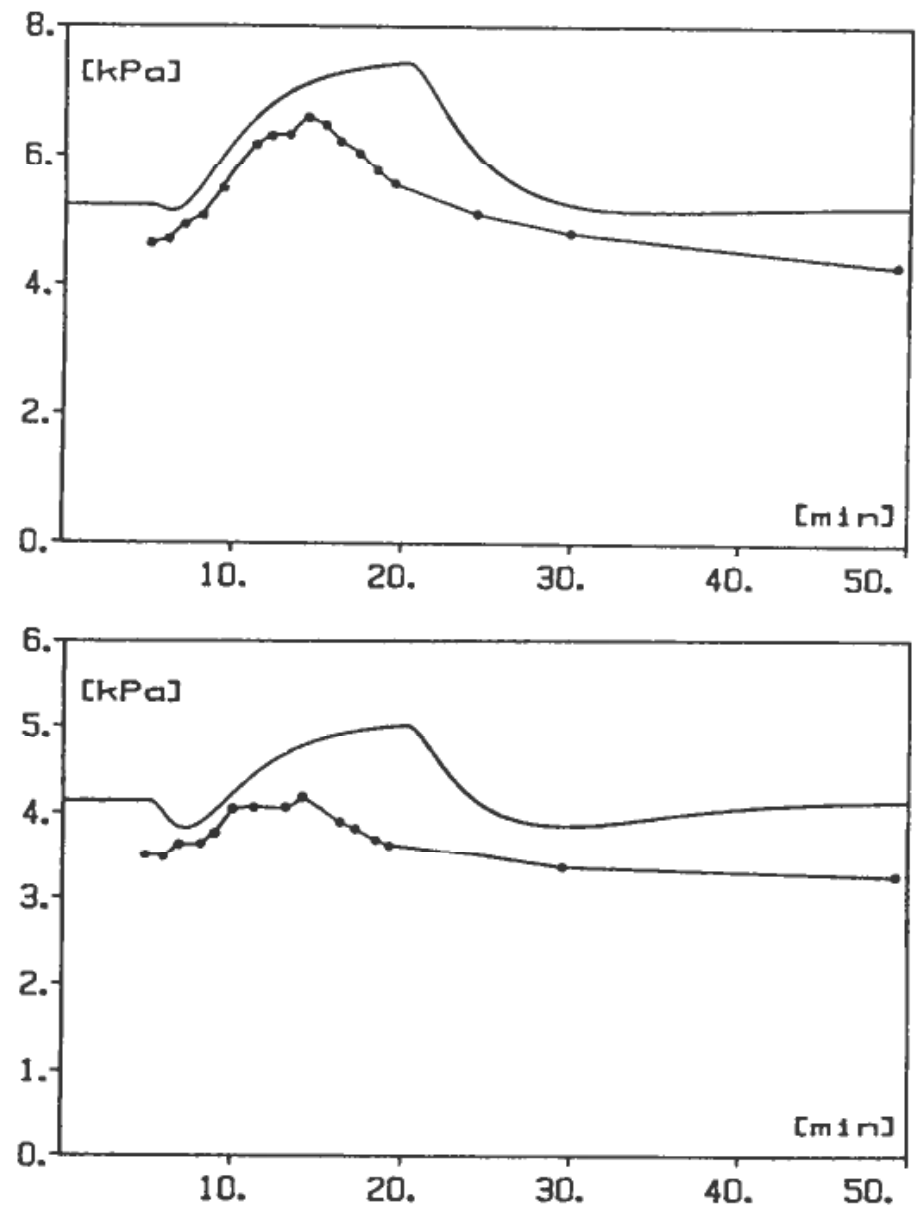

Figure 3. Responses of ventral (top) and dorsal (bottom) blood pressure after an intermittent increment in swimming speed from 5 until $20 \mathrm{~min}$. Dots: mean-pressure data calculated from Stevens and Randall (1967a). 

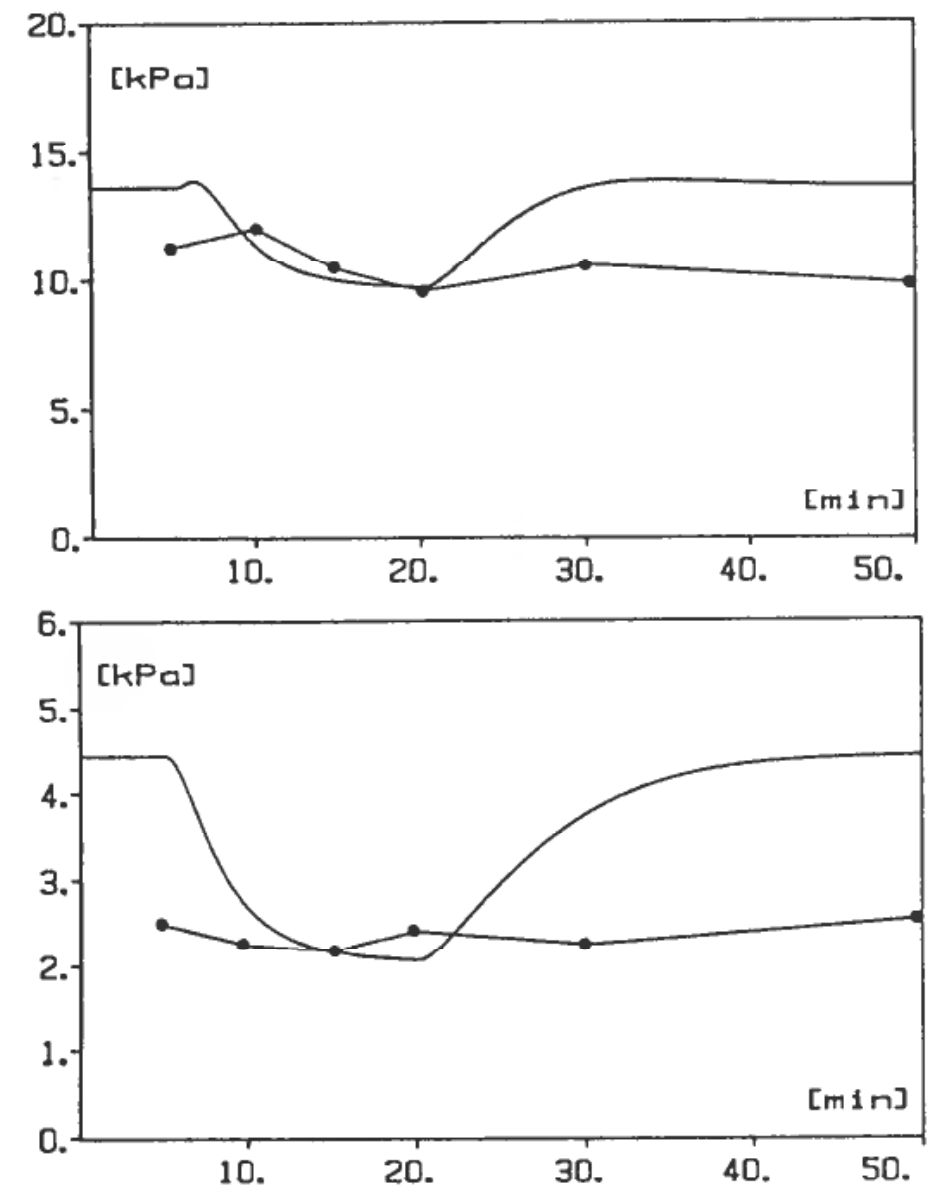

Figure 4. Responses of arterial (top) and venous (bottom) blood oxygen partial pressure after an intermittent increment in swimming speed from 5 until 20 min. Dots: data calculated from Stevens and Randall (1967b).

the activity, and overshoots at the beginning of recovery. The venous oxygen controller does not function well in the simulated case; the real response is far more stable. If the gain of the venous oxygen controller is increased, the venous oxygen variation decreases and the initial arterial overshoot becomes more pronounced. Thus, the controller gain found by static calibration is too low. This response may also point to a weakness in the proposed control structure, an illustration of the fact that dynamic experiments yield more information than static experiments, and the power of dynamic modelling in hypotheses testing.

Some of the numerical deviations between model and real fish are attributed to the different conditions for the static experiments used for model calibration and the dynamic experiment used for testing. In addition, the fish used in the dynamic experiment have a ventilation/blood flow ratio of about 100, an exceptionally high value, possibly indicating a weakness in the experimental procedures or measurement errors. The actual blood dissociation curve is not known, either for the fish used for static calibration or for those used in the cited dynamic experiment. Differences here will introduce errors into the partial pressure calculations. 


\section{Feeding behaviour}

The most important aspect of fish behaviour related to aquaculture is the feeding behaviour and possible hierarchical systems among the individuals. Most behavioural studies have been undertaken in various types of tanks and are therefore fairly representative for cultivated fish. However, most studies are based on only a few fish, whereas in aquaculture the fish density is very high. The few experiments on large collections of fish relate to growth rate differentiation, but there have also been some observations of hierarchical indicators such as spatial distribution and aggression/avoidance.

Using growth rate distributions over time is a slow way of estimating feeding behaviour, and they are not useful in practical aquaculture because under uncontrolled situations many other effects may influence growth differentiation. A model-based estimator will therefore be more appropriate as an aid in daily feeding management. For such applications a simple model of feeding behaviour was developed. This model is based on a combination of several probability functions along the lines presented in Balchen (1979).

\subsection{Appetite}

Appetite as used here is the short-term desire for food, often given as amount of feed ingested per time unit. In the long term, the appetite is controlled by a set point given by the central nervous system, depending on several factors. Long-term appetite is often called hunger. Appetite involves metabolic, neurophysical, and hormonal mechanisms and may be stimulated by metabolic factors such as levels of certain metabolites in the blood. In many cases the appetite is mainly dependent on stomach fullness $x$, which again is a function of amount of food previously eaten and stomach evacuation rate. For fish number $i$, this may be formulated as

$$
\dot{x}_{i}=V_{p} w_{p} / V_{s i}-a x_{i},
$$

where $x_{i} \in[0,1]$ is the fullness and $V_{p}$ the average food particle volume. The rate of particle ingestion $w_{p}$ and the coefficient of emptying rate $a$ are in this case both assumed to be independent of fish size. For a single fish fed ad libitum, $w_{p}$ is the maximum rate at which the fish can feed. The maximum stomach volume $V_{s i}$ is given by Grove $e t$ al. (1978) as

$$
V_{s i}=0 \cdot 075 W_{i}-0 \cdot 8,
$$

where $W_{i}$ is the whole fish weight.

A relationship between stomach fullness and appetite has been found for several species, including brown trout, rainbow trout, dab, sockeye salmon, and turbot. The data in Elliott (1975) and Grove et al. (1978) yield a sigmoid function for appetite vs. stomach fullness. A possible approximation to the mean of these data for fish number $i$ is the probability function

$$
p_{a i}= \begin{cases}0 \cdot 50-0 \cdot 57\left(x_{i}-0 \cdot 3\right) /\left(x_{i}-0 \cdot 2\right), & x_{i}>0 \cdot 3, \\ 0 \cdot 50+0 \cdot 67\left(0 \cdot 3-x_{i}\right) /\left(0 \cdot 4-x_{i}\right), & x_{i} \leqslant 0 \cdot 3 .\end{cases}
$$

\subsection{Food availability}

The food taken by fish depends on several factors, such as visual conditions, particle size and distribution, and confusion effects. Visual conditions include light intensity, 
water turbidity, and particle colour/background contrast. Fish usually forage most intensely at sunrise and sunset, when enough light is present to detect prey while at the same time the low light condition lowers the probability of the fish themselves being preyed upon. This may be modeled by a function of light intensity modulating the appetite. The distance at which food can be detected and the motivation to go for the food depend on particle size, as given by Wańkowski (1979). These results yield probabilities of food capture and ingestion/rejection, which combined provide a function for optimal feed particle diameter of $0.02 \times$ fish length. In aquaculture this is a well-known function, and particle size is chosen according to fish size. There is therefore no need to model this process.

The probability of a fish catching a food particle obviously depends on the density of particles in the water volume. However, assuming the feed rate to be appropriate, social interactions may be more important. Depending on the method of introducing the feed into the tank, the food particle distribution may vary throughout the water volume. Therefore a distribution index $\rho$ is introduced, being 1 for even distribution and 0 if all particles are concentrated at one point. When food is spatially concentrated, the fish will be attracted to this space, thus increasing the local fish density. This contributes to confusion, because the fish must use more of their attention to avoid other fish rather than fully concentrating on catching food. A confusion factor probability function is therefore included:

$$
p_{c}=\rho^{b}
$$

where $b>0$ is a constant. If the confusion depends on the length of free space in front of the fish, then $b=1 / 3$. If the confusion relates to the spatial angle of free view in front of the fish, $b=2 / 3$, and if it depends on free volume around the fish, $b$ will be equal to 1 . In the present model, $\bar{b}=0.4$ will be applied, corresponding to a dependency between the spatial angle and linear length cases.

\subsection{Social interaction}

Social status among salmonids is mainly dependent on size; large fish dominate over smaller fish. When few fish are kept in a tank, many species tend to develop a despotic hierarchy, where one fish dominates over all the others. At higher densities that are more relevant to aquaculture, however, linear hierarchies usually develop. In these hierarchies each individual dominates all others with lower rank, although aggression tends to occur between fish of nearly equal rank.

The hierarchical strength is believed to increase with appetite and concentration of food particles in space. When fish are well fed and food is evenly distributed, hierarchical effects may be fairly low, while the opposite may be the case of the fish are scantily fed and/or the feed is introduced by a point feeder. The appetite may be averaged over all individuals of the population. However, the appetite of the larger, potentially dominating, fish is most important for the hierarchical strength. Thus a weighted mean appetite can be computed as

$$
f_{a}=\frac{1}{W_{t}} \sum_{i=1}^{N} p_{a i} W_{i}
$$

where $W_{t}$ is the total weight of fish in the tank and $N$ the number of fish. 
The hierarchical strength dependence on food distribution can be formulated as

$$
f_{d}=\rho^{-c},
$$

where $c>0$ is a constant. The combined hierarchical effects from the averaged appetite and feed distribution can be mapped into a function expressing the probability for each fish to catch food depending on its rank in the hierarchy; for example,

$$
p_{h i}=\left(W_{i} / W_{N}\right)^{f_{a} f_{d}} \text {. }
$$

Here $W_{N}$ is the weight of the largest fish. Thus, when the hierarchical exponent $f_{a} f_{d}$ is 0 . there are no hierarchical effects, at $\mathbf{1}$ the hierarchical effect is proportional to weight, and at $\infty$ only the largest fish gets access to the food.
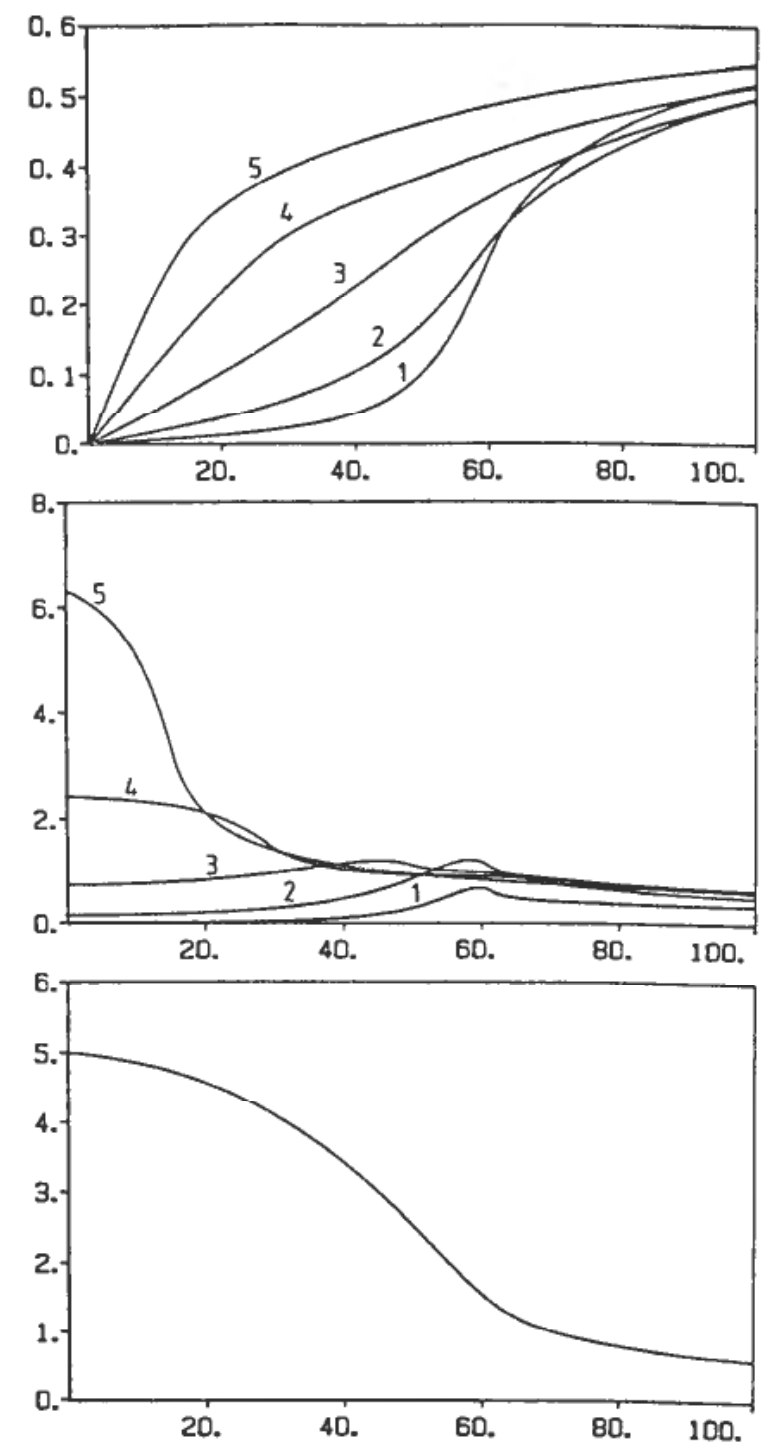

Figure 5. Feeding behaviour for five size classes, number 5 being the largest and number 1 the smallest. Top: stomach filling; middle: food taken, particles/minute/fish; bottom: hierarchical exponent. Time axis (abscissa) in minutes. 


\subsection{Model simulation}

The probability for a fish catching a particle is the product of the probability functions and the maximum feed rate,

$$
p_{t i}=w_{p} p_{c} p_{a i} p_{h i}
$$

Multiplying by particle size yields the volume of feed taken per time unit.

The above model was simulated to check if the behaviour was as expected. For this purpose a stock of 2000 fish with a normal size distribution, mean weight $30 \mathrm{~g}$, and standard deviation $5 \mathrm{~g}$ was used. A suitable food particle volume is $V_{p}=10 \mu \mathrm{L}$. The fish were aggregated into 21 size groups, each spanning $2 \mathrm{~g}$, ranging from 14 to $48 \mathrm{~g}$. The stomach filling for each group was modeled according to (17).

Figure 5 shows the results from a simulation with a feed distribution index of $\rho=0.2$. For clarity, only the size groups centred around (1) $14 \mathrm{~g}$, (2) $22 \mathrm{~g},(3) 30 \mathrm{~g}$, (4) $38 \mathrm{~g}$, and (5) $46 \mathrm{~g}$ are shown. All fish have empty stomachs when the simulation starts. One can see that the smaller fish must wait for the larger ones to become somewhat satiated before they get any significant access to the food. The hierarchical exponent $\left(f_{a} f_{d}\right)$ starts off well above 1 but stabilizes below 1 . The responses illustrate hierarchical effects that have often been observed in salmonids kept in tanks; however, it is hard to find quantitative data to compare them to. The crudest measure is the variation in total feed taken with time, but this does not give much information on the feed taken by each group or individual. One can speculate about videotaping and subsequent analysis by computers, or tagging each fish with tags transponding data on food capture. But both techniques are at present not realistic for large collections of fish.

\section{Conclusions}

The use of static descriptive experiments that are not based on models, which are common in biological sciences, limits the level of knowledge that can be achieved about biological systems. One ends up doing more and more experiments on the same theme to possibly obtain new curve-fitting parameters. Thus an important element of such research has become the various statistical methods used to fit lines to a few data points, an indication of having reached a wall. Several researchers in fish physiology are now considering whether explanatory mathematical models can be a means of removing this barrier. These models have much to offer an experimenter while scrutinizing the causes of the responses observed. If structured mathematical models were applied, a team of modelers and biologists would be able to do an impressive amount of work in fish physiology research. The models presented here represent a starting point for such efforts. With iterative experimentation and modeling, the models and knowledge will inevitably be improved above present standards. Although optimistic in this respect, we must point to the limits imposed on biological models - there is more to life, even in the simplest organisms, than a collection of physical and chemical formulas.

In addition to the publications that are referred to, the models presented here are based on information collected from several hundred publications. This puzzle work has several drawbacks, especially when the data and responses stem from experiments undertaken under different conditions, often on diverse fish species. Only a few experiments published in the literature are dynamic; the few that were found mainly treat gastric evacuation. This severely limits the knowledge neeeded to formulate dynamic models. Taking this into consideration, our results are rather impressive.

Several of our models can be tuned to obtain better agreement between model responses and real data. If more independent sets of data were available, one might tune 
the models to agree with one data set and then test the qualities of the model by comparing with other sets. However, data are sparse. We therefore chose to first calibrate the models against static data and pieces of knowledge and then compare results with real dynamic responses, using as little tuning as possible. At this stage of the modeling of fish phyisology, the most important goal is to verify the model structure. Numerical deviations are therefore less important than the qualities of the responses.

The digestive and absorptive processes of the fish are not well understood; the feedback mechanisms introduced here may be useful to improve the understanding of the gastric evacuation process. We will then have to find a high-quality model of the enzymes and chemistry of digestion and absorption. This will be essential to the evolution of fish growth models because classic descriptive experiments on growth vs. feeding do not yield much more knowledge than has already been acquired.

For the study of metabolic processes and hormones, a metabolic model combined with state estimation and experiments will be useful, especially as this method allows for dynamic experiments. Metabolic models like the one presented here are presently not useful in the modeling of growth, because the calculated growth rates mainly depend on the assumed nutrient absorption rates, not on the particular paths by which they are transformed in the metabolism. However, the consumption of oxygen calculated by metabolic models may be useful in practice. There is a trend toward lifelong culturing of salmon in land-based tanks. In this situation the fish are kept at very high densities, and extra oxygen must be introduced into the water to support the feeding respiration. In such a situation, metabolic models can be useful for optimal control of the oxygen injection.

The models of the blood/gill system will be useful in fish physiology research but have no immediate application in aquaculture.

Feeding behaviour models will also be beneficial in research. This method may enable behavioural researchers to get more knowledge out of their observations and growth rate dispersion experiments and use probability functions to formulate their theories. A better understanding of feeding behaviour may yield better procedures for feeding in aquaculture, thus allowing for more optimal feed utilization and lower weight dispersion.

\section{ACKNOWLEDGMENTS}

This work was supported by Siemens A/S Trondheim and a scholarship from the Norwegian Institute of Technology (OAO).

\section{REFERENCES}

BALCHEN, J. G. (1979). Modeling, prediction, and control of fish behavior, in Control and Dynamic Systems, Vol. 15, C. T. Leondes, Ed. (Academic, New York) pp. 99-146.

BALCHEN, J. G. (1989). Instrumentation, information and control in aquaculture, Aquaculture '89, World Aquaculture Society Congress, Los Angeles, Feb. 15, 1989.

Brett, J. R., and Groves, T. D. D. (1979). Physiological energetics, in Fish Physiology, Vol. 8, W. S. Hoar, D. J. Randall, and J. R. Brett, Eds. (Academic, Orlando, Fla.,) pp. 280-352.

ElliotT, J. M. (1975). Number of meals in a day, maximum weight of food consumed in a day and maximum rate of feeding for brown trout, Salmo trutta L., Freshwat. Biol., 5, 287-303.

From, J. and RASMUSSEN, G. (1984). A growth model, gastric evacuation, and body composition in rainbow trout, Salmo gairdneri Richardson, 1836. Dana 3, 61-139.

Grove, D. J., LoIzideS, L. G. and NotT, J. (1978). Satiation amount, frequency of feeding and gastric emptying rate in Salmo gairdneri. J. Fish Biol., 12, 507-516. 
Holmgren, S., Grove, D. J. and Fletcher, D. J. (1983). Digestion and the control of gastrointestinal motility, in Control Processes in Fish Physiology, J. C. Rankin, T. J. Pitcher, and R. Duggan, Eds. (Croom Helm, London), pp. 23-40.

JobLING, M. (1981). Mathematical models of gastric emptying and the estimation of daily rates of food consumption for fish. J. Fish Biol., 19, 245-257.

Jobling, M. (1987). Influences of food particle size and dietary energy content on patterns of gastric evacuation in fish: test of a physiological model of gastric emptying. J. Fish Biol., 30, 299-314.

Kenyon, C. J., MCKeEver, A., Oliver, J. A. and Henderson, I. W. (1985). Control of renal and adrenocortical function by the renin-angiotensin system in two euryhaline teleost fishes. Gen. Comp. Endocrinol. 58, 93-100.

KICENIUK, J. W. and JONES, D. R. (1977). The oxygen transport system in trout (Salmo gairdneri) during sustained exercise. J. Exp. Biol., 69, 247-260.

Machiels, M. A. M. and Henken, A. M. (1986). A dynamic simulation model for growth of the African catfish, Clarias gariepinus (Burchell 1822). I. Effect of feeding level on growth and energy metabolism. Aquaculture, 56, 29-52 (1986).

MALTE, H. and Weber, R. E. (1985). A mathematical model for gas exchange in the fish gill based on non-linear blood gas equilibrium curves. Resp. Physiol., 62, 359-374.

OLSEN, O. A. and BALCHEN, J. G. (1988). Simulation applied to state estimation in aquaculture. Proc. IMACS 12th World Congr. Sci. Comput. III, Paris, July 18-22, pp.655-659.

PIIPER, J. (1982). Respiratory gas exchange at lungs, gills and tissues: mechanisms and adjustments. J. Exp. Biol., 100, 5-22.

PIIPER, J. and SCHEID, P. (1984). Model analysis of gas transfer in fish gills in Fish Physiology, Vol. 10A, Gills, W.S. Hoar and D. J. Randall, Eds. (Academic, Orlando, Fla.), pp. 229-262.

Press, W. H., Flannery, B. P., Teukolsky, S. A. and Vetterling, W. T. (1989). Numerical Recipes in Pascal, (Cambridge Univ. Press, Cambridge).

RUOHONEN, K. (1986). Production planning and whole farm simulations in aquaculture, in Proc. IFAC Automation and Data Processing in Aquaculture, J. G. Balchen, Ed., Trondheim, Norway, 18-21 Aug. 1986, pp. 75-80.

Ruohonen, K. (1988). Production planning and whole farm simulations in aquaculture, Proc. IMACS 12th World Congr. Sci. Comput. III, Paris, July 18-22, pp. 660-662.

Schulz, A. R. (1978). Simulation of energy metabolism in the simple-stomached animal. $\boldsymbol{B r}$. $J$. Nutr., 39, 235-254.

Stevens, E. D. and RANDALL, D. J. (1967 a). Changes in blood pressure, heart rate and breathing rate during moderate swimming activity in rainbow trout. $J$. Exp. Biol., 46, 307-315.

SteVEnS, E. D. and Randall, D. J. (1967 b). Changes of gas concentrations in blood and water during moderate swimming activity in rainbow trout. J. Exp. Biol., 46, 329-337.

Sundby, A., Eliassen, K., Blom, A. and AasgaArd, T. (1991). Plasma insulin, glucagon, glucagon-like peptide and glucose levels in response to feeding, starvation and life long restricted feed ration in salmonids. Fish Physiol. Biochem., 9, 253-259.

TAYlor, W., Houston, A. H. and Horgan, J. D. (1968). Development of a computer model simulating some aspects of the cardiovascular-respiratory dynamics of the salmonid fish. J. Exp. Biol., 49, 477-493.

WAŃkowsKı, J. W. J. (1979). Morphological limitations, prey size selectivity, and growth response of juvenile Atlantic salmon, Salmo salar. J. Fish Biol., 14, 89-100. 